\title{
Análisis del marco regulatorio cubano sobre requisitos de gestión documental
}

\author{
Jorge del Castillo Guevara*
}

Artículo recibido:

7 de septiembre de 2020

Artículo aceptado:

26 de octubre de 2020

Artículo de revisión

\section{Resumen}

El Plan Nacional de Desarrollo Económico y Social para 2030 implica redimensionar la gestión documental de la Administración Pública y su correspondiente marco regulatorio, de manera que impulse prácticas informacionales responsables y transparentes. Se aplica el análisis documental en 322 fuentes legales y normativas cubanas respecto a la gestión documental. El análisis se realizó a partir de tres parámetros. Se presentan las deficiencias en cuanto a los requisitos de gestión documental en las regulaciones archivísticas y no archivísticas, así como los enfoques

* Facultad de Comunicación, Universidad de La Habana, Cuba guevara@fcom.uh.cu cuidarte312@gmail.com 
sobre la práctica profesional de gestión documental para el sector público. Se considera que estas son áreas de desarrollo esenciales para construir entornos informacionales que propicien la transparencia, la rendición de cuentas y la seguridad razonable en la función pública.

Palabras clave: Administración Pública; Cuba; Marco Regulatorio; Fuentes Legales y Normativas; Gestión Documental; Sistema Nacional de Archivos de la República de Cuba

Analysis of the Cuban regulatory framework on records management requirement

Jorge del Castillo Guevara

\begin{abstract}
The National Plan of Economic and Social Development for 2030 involves re-dimensioning the records management of the Public Administration and its corresponding regulatory framework, so as to promote responsible and transparent informational practices. Documentary analysis is applied in 322 Cuban legal and regulatory sources regarding records management. The analysis was carried out based on three parameters. The deficiencies in records management requirements in archival and non-archival regulations are presented, as well as approaches to professional records management practice for the public sector. These are considered to be essential development areas for building informational environments that foster transparency, accountability, and reasonable security in the civil service.
\end{abstract}

Keywords: Public Administration; Cuba; Regulatory Framework; Legal and Regulatory Sources; Records Management; National Archives System of the Republic of Cuba 


\section{INTRODUCCIÓN}

Cuba cambia. Cada día se hace más ineludible la idea de un país con un ejercicio de gobierno más abierto, transparente e inclusivo, con un enfoque orientado a la rendición de cuentas y a un mayor control social sobre las instituciones de la Administración Pública (AP), y por eso hoy se habla de comunicación con enfoques de derecho, de equidad y de gestión del conocimiento.

Una nueva constitución ha ratificado el compromiso del país con el desarrollo de una sociedad de la información y del conocimiento "centrada en la persona, integradora y orientada al desarrollo sostenible, en la que todos pueden crear, consultar, utilizar y compartir la información y el conocimiento en la mejora de su calidad de vida" (Constitución, 2019: 3), en virtud de un modelo de comunicación pública más abierto.

El cumplimiento del mandato constitucional implica que la Administración Pública Cubana (APC) cuente con buenas prácticas de gestión documental (GD) para que las organizaciones puedan realizar sus procesos de trabajo en un contexto razonablemente seguro, sobre la base de información veraz. En auditorías recientes realizadas por la Contraloría General de la República (CGR) se han señalado múltiples incidencias relacionadas con la "falta de fiabilidad de la documentación (información) primaria o la inexistencia de la misma", así como la "inefectividad en los mecanismos de información" (Rivero Acosta, 2017).

Para contar con efectivos sistemas de gestión documental (SGD) en la APC, se requiere un marco regulatorio y normativo que reconozca las mejores prácticas en este sentido, es decir, un marco regulatorio que contenga requisitos que aseguren una gestión coherente de documentos de archivo (en adelante documentos) para que las organizaciones sean transparentes y rindan cuentas de sus actos con información veraz.

\section{Metodología}

Se utilizó como método de investigación el análisis documental, el cual se aplicó sobre las fuentes legales y normativas recopiladas con base en las siguientes interrogantes:

- ¿Qué tipo de requisitos de GD contiene el marco regulatorio y normativo cubano?

- ¿Existe una integración coherente entre las regulaciones archivísticas y el resto de las fuentes legales y normativas que conforman el marco regulatorio y normativo cubano? 
- ¿El marco regulatorio cubano presenta un enfoque de integración sobre la GD o de segmentación profesional respecto a ésta y la administración de archivo?

Los parámetros para el análisis fueron los siguientes:

- Tipos de requisitos de GD. Entendido como "una restricción, demanda, condición, necesidad o parámetro que debe ser identificado o satisfecho usualmente dentro de un marco de tiempo o como un prerrequisito" (InterPARES 2 Project, 2008: 43). A partir de este parámetro se identificaron los requisitos que aplican en los procesos de GD que establece la norma ISO 15489-1 (ISO, 2016).

- Requisitos de creación. La creación es el momento en el que tienen lugar las acciones a partir de las cuales se generan los documentos, así como la representación de tales acciones sobre los propios documentos (Reed, 2005). La adecuada gestión de la creación resulta crucial porque establece una conexión entre las funciones y operaciones de trabajo con los documentos resultantes (Dikopoulou y Mihiotis, 2011). Los requisitos de creación son aquellos que se relacionan con los momentos de la producción del documento durante la transacción, antes de su captura en el sistema (ISO, 2016).

- Requisitos de captura. La captura ocurre cuando se han definido los documentos que se necesitan mantener y gestionar a lo largo del tiempo (ISO, 2016). Durante la captura se genera un ítem de información que es evidencia de las actividades de una unidad organizativa. Por medio de este proceso el documento alcanza su solidez como evidencia de acciones (Reed, 2005). Los requisitos de captura son aquellos que se orientan a la incorporación del documento al sistema.

- Requisitos de clasificación. La clasificación es el proceso en el que los documentos se vinculan con su contexto de negocio (función, actividad o proceso de trabajo) y con su agregación documental, a partir de un sistema de clasificación (ISO, 2016). Los requisitos de clasificación son aquellos que tienen que ver con la clasificación archivística.

- Requisitos de control de acceso. El acceso es uno de los aspectos medulares para la archivística contemporánea por su implicación en el gobierno abierto, la rendición de cuentas, la transparencia, el derecho a la información y el conocimiento y la justicia social, entre otros escenarios. Como asevera Menne-Haritz (2001), el uso es 
lo único que justifica la existencia de los archivos. La norma ISO 15489-1 define acceso como "derecho, oportunidad y medios para localizar, usar o recuperar información” (ISO, 2016: 1). Desde la propia norma se puede comprender el control de acceso como uno de los mecanismos para proveer y/o restringir el acceso a documentos, a partir de determinadas reglas y derechos de acceso establecidos de antemano. Se entenderá como requisitos de control de acceso aquellos que regulan el acceso a los documentos.

- Requisitos de almacenamiento. El almacenamiento comprende las medidas para la protección de los documentos contra posible acceso, modificación y destrucción no autorizada, así como pérdida, robo o desastre (ISO, 2016). Los requisitos de almacenamiento se refieren a aquellos que condicionan la protección de los documentos en todo momento.

- Requisitos de migración. La migración es un proceso que implica un alto riesgo para los documentos por la posible alteración de su integridad física y composición lógica (Duranti, 2001). Es por ello que resulta primordial controlar y asegurar la integridad y autenticidad de los documentos que se someten a procesos de migración. Los requisitos de migración son aquellos que aseguran el mantenimiento de los elementos que conforman el documento y sus metadatos asociados durante este proceso.

- Requisitos de disposición. Se refiere al proceso en el que se determina el periodo de tiempo que se deben retener los documentos y sus metadatos asociados, abarca su eliminación y transferencia hacia una custodia diferente. Los requisitos de disposición son las restricciones, demandas, condiciones, necesidades o parámetros que deben ser cumplidos para la retención, eliminación y transferencia de documentos.

- Relación entre las regulaciones archivísticas y no archivísticas. En este parámetro se estudió si existe una integración armónica entre ambas regulaciones. Se consideran regulaciones archivísticas aquellas que formalizan la GD en el país; las no archivísticas se refieren al resto de fuentes legales que conforman el marco regulatorio cubano.

- Enfoque sobre la práctica profesional de GD (inclusiva o exclusiva de la administración de archivo). Desde las perspectivas más contemporáneas, se defiende un enfoque de integración teórica y profesional sobre la GD que abarca las prácticas de administración de archivo. Como asevera McKemmish (2017: 122-123): 
La gestión documental abarca una gama de procesos y actividades de archivo, archiving processes y de recordkeeping entrelazados, llevados a cabo por los records manager y archivist para fines actuales, regulatorios e históricos de recordkeeping. Estos propósitos incluyen los roles que desempeña la gestión documental, a través del espacio y el tiempo en la gobernanza y la rendición de cuentas, el recuerdo y el olvido, la configuración de la identidad y la diseminación de fuentes de información de valor agregado. En términos clasificatorios, "recordkeeping" en este uso incluye el records management y la archival administration.

A partir de este parámetro se identifica si las regulaciones y normas cubanas asumen un enfoque que separa la gestión documental de la administración de archivo, o si por el contrario asumen una práctica profesional de integración entre estas nociones.

El estudio abarcó 322 fuentes legales (por ejemplo, Leyes, Decretos, Resoluciones) y normas técnicas (emitidas por la Oficina Nacional de Normalización) hasta el cierre de mayo de 2020. La selección de las fuentes se realizó a partir de seis áreas funcionales que son comunes a todas las instituciones de la APC: gestión económica y financiera; gestión del capital humano; gestión de información, tecnológica y de comunicación; seguridad y protección; defensa y defensa civil, e investigación e innovación tecnológica. En el Anexo se encuentra un índice con las fuentes legales y normativas citadas en este material.

\section{UN ACERCAMIENTO A LA GESTIÓN DOCUMENTAL EN EL CONTEXTO INTERNACIONAL}

La archivística ha experimentado, desde la década de los 80 , todo un proceso de reconceptualización teórica, por lo que autores como Bearman (1994), Cook (1997, 2001, 2013), Duranti (2001), Gilliland-Swetland (2000), McKemmish (2017), Menne-Haritz (2001), Upward (1996, 1997), entre otros, han delineado los rasgos de lo que se ha conocido como paradigma poscustodial (Ribeiro, 2017). En los debates más contemporáneos en torno a la archivística y la gestión documental se destacan cuestiones como:

- La reconceptualización de la noción de documento, de considerarlo como un objeto de naturaleza fija a comprenderlo como algo mutable, siempre en estado de formación.

- El énfasis en la naturaleza probatoria, contextual y transaccional sobre el documento.

- Un enfoque de integración entre la archivística y la gestión documental.

- La relevancia del acceso a los documentos. 
- La necesidad de especificar requisitos para gestionar documentos de calidad.

- Un enfoque de los sistemas y procesos de gestión documental de cara a la gobernanza de la información.

Sobre este último aspecto es posible afirmar que los enfoques sobre la práctica profesional de la gestión documental se transformaron como resultado de la "revolución de la información electrónica" (Bearman, 1994) y experiencias internacionales que pusieron al descubierto las consecuencias de malas prácticas documentales. Si bien en la década de los 50 del pasado siglo la gestión documental se orientó a problemas de ineficiencia y mala administración, para 1990 comienza a relacionarse con fraude y corrupción (McKinnon, 1994), y en los inicios del siglo XXI se pone de manifiesto una clara relación con la gestión de riesgos (Committee of Sponsoring Organizations of the Treadway Commission, 2004; ISO, 2014; Lemieux, 2004; 2010) y la gobernanza de la información (ARMA International, 2014).

Las organizaciones contemporáneas se caracterizan por estructuras más horizontales, flexibles, policéntricas, matriciales y conectadas en redes de trabajo (Foscarini, 2010); por la penetración de medios sociales, lo cual ha impactado en las relaciones de confianza entre la administración y la ciudadanía (Evans, Franks y Chen, 2018); por un entorno laboral cada vez más desmaterializado y desterritorializado, con un tipo de documento que existe en red, de múltiples procedencias, extrainstitucional, transinstitucional y transjurisdiccional (Gilliland-Swetland, 2014).

Tales características, en adición a diversos escándalos internacionales de corrupción relacionados con prácticas documentales ineficientes, tuvieron importantes implicaciones para la GD en los ámbitos normativos y legislativos (Mena Mugica y Castillo Guevara, 2018).

En el ámbito normativo, el Comité Técnico TC 46 "Información y Documentación”, Subcomité SC 11 "Archivos/Gestión Documental”, de la Organización Internacional de Normalización, ha publicado un amplio conjunto de normas técnicas que conforman un marco de buenas prácticas en este sentido. La ISO 15489 Information and documentation - Record management ha sido una de las más relevantes. Su primera versión se publicó en 2001 y fue elaborada a partir de la norma australiana de 1996 AS 4390 Records Management. La ISO 15489 consta de dos partes, la primera establece aspectos generales de la GD (ISO, 2001a) y la segunda es un reporte técnico para aplicar la primera parte (ISO, 2001b). En 2016 se publicó una nueva versión, la ISO 15489-1 Information and Documentation - Records Management - Part 1: Concepts and Principles que modifica y cancela la anterior (ISO, 2016). Esta norma establece 
los conceptos, principios y procesos para una adecuada creación, captura y gestión de documentos archivísticos que son aplicables en cualquier contexto organizacional. Además, ha contribuido a redimensionar el enfoque sobre el rol de los gestores documentales hacia una perspectiva de gestión más activa y estratégica, orientada a la gobernanza, la gestión de la calidad y la auditoría (Joseph, Debowski y Goldschmidt, 2012).

Otras normas de GD han sido publicadas, como la familia de normas ISO 30300 Information and documentation - Management system for records, las cuales ofrecen herramientas y métodos para la implementación, desarrollo y mejora de un efectivo SGD. Otros aspectos, como la gestión de metadatos, el análisis de procesos de trabajo, la identificación y evaluación de riesgos y la preservación digital, también han sido sistematizados mediante normas técnicas ISO.

En el ámbito legislativo, la Public Company Accounting Reform and Investor Protection Act, popularizada como Sarbanes-Oxley Act, regulación de Estado Unidos, tuvo repercusiones significativas para la GD en las instituciones públicas de ese país respecto a la preservación, retención y destrucción de evidencia documental. Otras herramientas encaminadas a la gobernanza corporativa, la transparencia y la rendición de cuentas (Mat Isa, 2009) han sido desarrolladas en otros países para reforzar sus sistemas de control interno.

Lo anterior evidencia la incorporación de requisitos de GD en el marco regulatorio para que las organizaciones puedan gestionar documentos de calidad.

\section{LA ADMINISTRACIÓN PÚBLICA CUBANA ACTUAL Y SU MARCO REGULATORIO}

Cuba se encuentra inmersa en un proceso de actualización de su modelo económico y social. Las amplias reformas que se llevan a cabo para el sistema de dirección y gestión pública han estado determinadas por la introducción de criterios como el fortalecimiento de la institucionalidad y la mayor transparencia gubernamental (Marqueti Nodarse, 2018). Todo ese proceso ha implicado, a su vez, una profunda actualización de su marco regulatorio y normativo.

En este trabajo se entiende al sector de la AP como una organización compleja que tiene la finalidad de gestionar el accionar del Estado, de cara a la satisfacción de las necesidades de la sociedad, sobre la base de un régimen jurídico particular (Cárdenas Travieso y Tabares Neira, 2018).

El Estado, por su parte, se comprende como "una forma de organización social, política soberana y coercitiva, formada por un conjunto de instituciones que tienen el poder de regular la vida nacional y que poseen la autoridad 
y potestad para establecer las normas que regulan una sociedad" (Tamayo Pineda y Fernández Estrada, 2018: 11).

La estructura central del Estado cubano es la siguiente:

- Asamblea Nacional del Poder Popular (ANPP). Es el órgano supremo del poder del Estado y está integrado por diputados, según el procedimiento que se establece en la ley, y cuenta con un presidente, un vicepresidente y un secretario. Se considera el único órgano en la república que tiene potestad constituyente y legislativa, y es elegida por cinco años.

- Consejo de Estado (CE). Se trata de un órgano colegiado, responsable ante la ANPP, a la cual le rinde cuentas acerca de sus actuaciones. Asimismo, representa a la ANPP entre periodo de sesiones.

- Presidente y Vicepresidente. El Presidente de la República es el Jefe del Estado y es elegido por la ANPP, a la que rinde cuentas de su gestión. El Vicepresidente cumple las atribuciones que le asigna el Presidente de la República y lo sustituye, temporalmente, en caso de ausencia, enfermedad o muerte. Son elegidos de igual modo por la ANPP.

- Consejo de Ministros (CM). Constituye el Gobierno de la República y es el órgano ejecutivo y administrativo de mayor jerarquía. El CM está conformado por el Primer Ministro, los Viceprimeros Ministros, los Ministros, el Secretario y otros miembros que se determinen por la ley.

- Comité Ejecutivo (CEj). Está integrado por el Primer Ministro, los Viceprimeros Ministros, el Secretario. Asimismo, el Presidente de la República puede determinar, además, otros miembros que conformen el CEj. Decide sobre cuestiones atribuidas al CM durante el periodo comprendido entre una reunión y otra.

- Administración Central del Estado (ACE). Está conformada por los ministerios e institutos que conforman los Organismos de la Administración Central del Estado (OACE).

- Tribunales de justicia. Son un sistema de órganos del Estado con independencia funcional. El Tribunal Supremo Popular (TSP) y el resto de los tribunales ejercen la función de impartir justicia a nombre del pueblo.

- Fiscalía General de la República (FGR). Este órgano ejerce el control sobre la investigación penal, así como el ejercicio de la acción penal pública en representación del Estado, y tiene independencia funcional. También vela por el cumplimiento de lo establecido en la constitución y el resto de las disposiciones legales.

- Contraloría General de la República (CGR). Este órgano del Estado vela por la administración correcta y transparente de los fondos públicos 
y el control de la gestión administrativa. También tiene independencia funcional y se subordina al Presidente de la República.

La producción jurídica cubana es facultativa de los órganos del Estado. La ANPP emite las regulaciones jurídicas de mayor rango en el ordenamiento jurídico cubano, como son las Leyes. En periodos de intersecciones, el CE representa a la ANPP y al Estado para fines nacionales e internacionales y tiene la facultad de emitir Decretos Ley con rango de Ley, los que podrán ser o no ratificados por la Asamblea.

El CM tiene la facultad de emitir Decretos que firma el Jefe del Gobierno y del Estado (Primer Ministro) y adoptar acuerdos. El CM y su CEj también dictan Reglamentos, que están orientados a regular una norma. Los ministros y otros directivos de OACE están facultados para dictar Resoluciones para regular el funcionamiento hacia el interior de cada sector, aunque se reconocen aquellas que se realizan de manera conjunta, entre dos o más ministerios. Otros órganos como la FGR y el TSP también tienen facultades para emitir regulaciones, aunque sólo en el marco de sus competencias.

En el ordenamiento jurídico cubano existe una jerarquía en cuanto a las regulaciones jurídicas. Según este orden, la Ley es la norma de mayor rango del Estado. El Decreto Ley es la disposición jurídica que emana del CE y tiene un rango inferior a la Ley. Los Decretos y los Acuerdos son emitidos por el CM y su CEj. En un nivel inferior se encuentran las Resoluciones emitidas por los ministros y jefes de los OACE. Todas éstas se subordinan a la Constitución de la República de Cuba.

\section{Resultados Del ANÁLisis DEL MARCO REgUlATORIO CUBANO DESDE LA PERSPECTIVA DE GD}

Los tipos de fuentes estudiadas y la cantidad por cada tipo se muestran en la Tabla 1. Para su recopilación se establecieron seis áreas funcionales comunes para todas las instituciones de la APC. Las áreas funcionales definidas y la cantidad de fuentes identificadas por cada una se presentan en la Tabla 2. En la Tabla 3 se presenta la cantidad de fuentes identificadas por organismo. En la Tabla 4 se advierte la cantidad de fuentes archivísticas y no archivísticas estudiadas. 


\begin{tabular}{|l|r|}
\hline \multicolumn{1}{|c|}{ Tipo de fuentes legales y normativas } & \multicolumn{1}{c|}{ Cantidad } \\
\hline Documento Constitucional & 1 \\
\hline Leyes & 22 \\
\hline Decretos Ley & 26 \\
\hline Decretos & 19 \\
\hline Resoluciones & 228 \\
\hline Resoluciones conjuntas & 5 \\
\hline Instrucciones & 7 \\
\hline Directivas & 2 \\
\hline Acuerdos & 6 \\
\hline Normas técnicas & 6 \\
\hline
\end{tabular}

Tabla 1. Cantidad de fuentes identificadas por cada tipo

\begin{tabular}{|l|r|}
\hline \multicolumn{1}{|c|}{ Áreas funcionales } & \multicolumn{1}{c|}{ Cantidad } \\
\hline Gestión económica y financiera & 85 \\
\hline Gestión del capital humano & 68 \\
\hline Gestión de información, tecnológica y de comunicación & 55 \\
\hline Seguridad y protección & 59 \\
\hline Defensa y defensa civil & 42 \\
\hline Investigación e innovación tecnológica & 13 \\
\hline
\end{tabular}

Tabla 2. Cantidad de fuentes legales y normativas identificadas por áreas funcionales

\begin{tabular}{|l|r|}
\hline \multicolumn{1}{|c|}{ Organismo } & \multicolumn{1}{c|}{ Cantidad } \\
\hline Asamblea Nacional del Poder Popular (ANPP) & 23 \\
\hline Consejo de Estado (CE) & 50 \\
\hline Consejo de Ministros (CM) & 46 \\
\hline Ministerio de Ciencia, Tecnología y Medio Ambiente (CITMA) & 30 \\
\hline Ministerio de Economía y Planificación (MEP) & 22 \\
\hline Ministerio de Finanzas y Precios (MFP) & 54 \\
\hline Ministerio del Comercio Exterior y la Inversión Extranjera (MINCEX) & 5 \\
\hline Ministerio de Comercio Interior (MCI) & 4 \\
\hline Ministerio de Trabajo y Seguridad Social (MTSS) & 23 \\
\hline Ministerio de la Informática y las Comunicaciones (MIC) & 21 \\
\hline Ministerio de Energía y Minas (MINEM) & 4 \\
\hline
\end{tabular}




\begin{tabular}{|l|r|}
\hline Ministerio del Interior (MININT) & 3 \\
\hline Ministerio de las Fuerzas Armadas Revolucionarias (FAR) & 9 \\
\hline Contraloría General de la República (CGR) & 4 \\
\hline Banco Central de Cuba (BCC) & 11 \\
\hline Estado Mayor Nacional de la Defensa Civil (EMNDC) & 7 \\
\hline Oficina Nacional de Normalización (ONN) & 6 \\
\hline
\end{tabular}

Tabla 3. Cantidad de fuentes legales y normativas por organismo

\begin{tabular}{|l|r|}
\hline \multicolumn{1}{|c|}{ Clasificación de fuentes legales y normativas } & Cantidad \\
\hline Fuentes archivísticas & 320 \\
\hline Fuentes no archivísticas & 2 \\
\hline
\end{tabular}

Tabla 4. Cantidad de fuentes legales y normativas según su clasificación en archivísticas y no archivística

La mayor cantidad de fuentes se concentra en las resoluciones, las cuales representan $71 \%$ del total analizado. Asimismo, el área funcional predominante, en relación con la cantidad de fuentes, es la gestión económica y financiera, para $26 \%$; seguida de gestión del capital humano, con $21 \%$. El organismo de mayor número de fuentes analizadas fue el Consejo de Estado, con una representación de $15 \%$. Las fuentes regulatorias archivísticas analizadas fueron el Decreto Ley (DL.) 265/09 "Del Sistema Nacional de Archivos de la República de Cuba" y la Resolución (R.) 41/09 del CITMA "Lineamientos para la Conservación de las Fuentes Documentales".

\section{Resultado del análisis por parámetros}

\section{Tipos de requisitos de gestión documental}

En relación con los requisitos de creación y de captura, no se encontraron en el DL. 265/09. En las disposiciones jurídicas no archivísticas se especifican los actos que deben ser documentados, aunque no para todos los casos. Los tipos documentales más frecuentes fueron informes, actas, expedientes, certificaciones, contratos, planes, registros, procedimientos y modelos establecidos por los OACE. También se hizo recurrente la presencia de requisitos sobre la definición del contenido de determinados documentos en el momento de la creación y captura. Este tipo de requisitos aparece, con mayor incidencia, en los documentos respecto a la gestión económica y financiera y a la gestión del capital humano (por ejemplo, la R. 11/07 del MFP "Pone en vigor los datos de 
uso obligatorio que se tendrán en cuenta al momento de diseñar los modelos del subsistema de inventarios" y la R. 29/14 del MTSS "Aprobar los informes, modelos y registros primarios a emplear por las entidades para cumplir lo establecido en el Reglamento del Código”). No obstante, la presencia de requisitos para la creación y captura de documentos archivísticos en aquellas fuentes relacionadas con el resto de las áreas funcionales resultó insignificante. Esta carencia pudiera afectar la capacidad de las instituciones para asegurar la confiabilidad de los documentos archivísticos, cualidad ésta que se relaciona con el momento de la creación.

Respecto a los requisitos de almacenamiento, se identificaron mayoritariamente en la R. 41/09 del CITMA. En esta resolución resaltan los requisitos sobre las características físicas de las instalaciones y medios de almacenamiento de documentos en diferentes soportes. Además, establece recomendaciones generales para su conservación. Las recomendaciones incluyen el cumplimiento de normas para la prevención contra incendios, contar con planes de conservación preventiva y contra desastres, control de vectores, entre otros aspectos.

Esta regulación presenta un abanico amplio de requisitos en este rubro, de gran utilidad para el almacenamiento seguro de la documentación archivística de los OACE. Sin embargo, resulta necesario advertir la marcada orientación de sus requisitos hacia las características y tratamiento de los soportes tecnológicos, lo cual puede estar relacionado con la concepción de los documentos archivísticos como objetos físicos. Esta visión no tiene en cuenta la dimensión lógica de estos documentos, puesto que los elementos que los estructuran (contenido, contexto y estructura) son lógicos más que físicos, con independencia de si se encuentran en papel o en soporte digital.

En el resto de las fuentes revisadas (regulaciones no archivísticas) se observaron casos muy específicos, como el de la R. 60/11 de la CGR "Normas del Sistema de Control Interno", que hace hincapié en los mecanismos de seguridad para el almacenamiento de la información oficial contable y estadística de los órganos, organismos, organizaciones y demás entidades. El Decreto (D.) 325/14 "Reglamento de la Ley de la Inversión Extranjera" establece la obligación por parte de los inversionistas de garantizar el adecuado almacenamiento, custodia y conservación de la documentación técnica. En ninguna de las regulaciones no archivísticas estudiadas se hace referencia a la R. 41/09 del CITMA.

En el ámbito de la seguridad informática, la Norma cubana NC ISO/IEC 17799 del 2007 Tecnología de la Información - Código de Buenas Prácticas para la Gestión de la Seguridad de la Información menciona el deber de establecerse procedimientos para el almacenamiento de la información con la finali- 
dad de garantizar su protección frente al mal uso o divulgación no autorizada (ONN, 2007).

En relación con los requisitos de disposición, el DL. 265/09 establece las funciones de la Comisión Nacional de Valoración Documental, que es la entidad que aprueba las propuestas de conservación y eliminación de documentos en las instituciones que pertenecen al Sistema Nacional de Archivos de la República de Cuba (SNARC) y también determina las funciones de las comisiones centrales de valoración en las instituciones.

En las regulaciones no archivísticas se advirtió, en repetidas ocasiones, la existencia de requisitos sobre la retención de documentos en los archivos de oficinas por el periodo de cinco años o mientras el documento mantenga su vigencia administrativa. Sin embargo, no se especifica qué ocurre con esa documentación pasado ese tiempo (algunos ejemplos son la R. 20/09 del MFP “Sobre Faltantes, Pérdidas y Sobrantes de Bienes”, y el D. 326/14 del CM "Reglamento del Código de Trabajo"). Se observó sólo un caso, el de la R. 54/05 del MFP "Elementos generales que deben contener los documentos normativos sobre contabilidad en las entidades, como parte del sistema de control interno", que preceptúa la definición de los plazos de retención de los documentos (aquí se refiere a documentos contables) una vez que culmine su vigencia administrativa. También se identificaron requisitos que establecen la retención permanente de determinados documentos relativos a la gestión del capital humano, como ocurre con los expedientes personales de los cuadros, que se mantienen en los respectivos centros laborales, según el DL. 196/99 "Sistema de Trabajo con los Cuadros del Estado y el Gobierno".

La eliminación documental se trata en las regulaciones jurídicas desde varias aristas. Se advirtieron requisitos relacionados con el momento en el que los documentos deben ser eliminados, como en la R. 1/04 del MFP y MIC "Requisitos para los Sistemas Contables - Financieros soportados sobre las tecnologías de la información”. Esta regulación estipula que los programas, ficheros y datos de los sistemas contables financieros, incluyendo las copias de respaldo, deben ser eliminados inmediatamente después de terminar la necesidad de su uso. Desde otra arista, el DL. 370/18 "Sobre la informatización de la sociedad cubana" regula que la destrucción dolosa de datos e información, así como de sistemas de información y comunicación de servicios públicos, sociales y administrativos, se consideran contravenciones asociadas a las TIC. Al mismo tiempo, la Ley 62/87 "Código penal” considera la destrucción de documentos en perjuicio del interés nacional o de una persona como un motivo para la aplicación de sanción de privación de libertad.

En el resto de los casos se hace referencia a la eliminación de documentos desde un enfoque preventivo, o sea, ante posibles desastres naturales $\mathrm{u}$ 
otras incidencias causadas por la manipulación intencional de personas. En relación con lo anterior, el DL. 199/99 "Sobre la Seguridad y Protección de la Información Oficial” prevé la destrucción de la información oficial en situaciones excepcionales y otras que puedan poner en riesgo su seguridad y protección.

Sin embargo, para la mayoría de los documentos que se especifican en las fuentes legales revisadas, no se prevén requisitos específicos sobre su retención y eliminación. Esto supone un alto riesgo por la posibilidad de eliminar documentos de forma indiscriminada en las instituciones de la AP, lo cual conlleva a que las instituciones no cuenten con evidencia suficiente para rendir cuenta de sus actos, delimitar responsabilidades o enfrentarse a posibles litigios.

Respecto a los requisitos de control de acceso, resulta necesario apuntar que el acceso a la información y el conocimiento es uno de los aspectos a los que mayor atención se le ha prestado en los últimos tiempos en las nuevas regulaciones aprobadas, como resultado de las transformaciones del modelo económico y social que se viven en Cuba. En la reciente Constitución de la República se dispone que todas las personas tienen derecho a solicitar y recibir información veraz, objetiva y oportuna de parte del Estado (Constitución, 2019). El Plan Nacional de Desarrollo Económico hasta 2030 insiste en fortalecer, entre otros aspectos, el control social y el acceso a la información de la AP, con el propósito de garantizar la rendición de cuentas, la calidad y eficacia de las políticas públicas. Asimismo, la Conceptualización del Modelo Económico y Social Cubano de Desarrollo Socialista destaca que la información, la comunicación y el conocimiento constituyen bienes públicos de derechos ciudadanos (Partido Comunista de Cuba, 2016).

Lo anterior contrasta con la regulación jurídica más importante del SNARC, el DL. 265/09, que sólo hace referencia a determinadas situaciones que condicionan la restricción del acceso. En relación con los fondos y colecciones del Archivo Nacional, el decreto supedita su acceso a la autorización del director del archivo, previa solicitud por escrito del interesado. Esto socava el derecho de libre acceso a la información que forma parte de la memoria histórica del país, y condiciona una práctica archivística desfavorable respecto al acceso. Respecto a los archivos institucionales, este Decreto Ley no prevé entre las funciones de los archivos centrales el servicio a usuarios internos o externos, por lo que no quedan esclarecidos los mecanismos para garantizar el acceso a la información de la AP.

En las regulaciones no archivísticas se encontraron pocos requisitos sobre el control de acceso. Los más frecuentes se orientan a la protección de información y documentos frente al acceso no autorizado, como se pudo advertir en algunas disposiciones jurídicas contables, de seguridad informática 
y las que se relacionan con la información oficial (por ejemplo, R. 1/04 del MFP y MIC; el D. 360/19 del CM "Sobre el establecimiento de la seguridad de las tecnologías de la informatización y la comunicación y la defensa del ciberespacio nacional”; DL. 199/99 del CE). Desde un enfoque centrado en la protección del patrimonio, el D. 118/83 "Reglamento para la Ejecución de la Ley de Protección al Patrimonio” establece el libre acceso y reconocimiento de cualquier bien que integre el Patrimonio Cultural, a fin de facilitar la labor de su salvaguarda y conservación por la Dirección de Patrimonio Cultural del Ministerio de Cultura.

Por otra parte, en el resto de las regulaciones jurídicas, el acceso se trata de manera muy específica; por ejemplo, la R. 11/12 del MTSS "Aprobar el cargo de Especialista Superior en Cuadros" hace referencia a los técnicos de cuadros y su función de dar acceso a los expedientes de cuadros a los cuadros, especialistas y técnicos debidamente autorizados. La R. 49/09 del MTSS "Calificador ramal de cargos técnicos de la Actividad de Ciencia e Innovación Tecnológica" se refiere a los técnicos en servicios de información que aseguran el acceso a las fuentes de información. Otro ejemplo es la norma NC ISO 45001 Sistema de Gestión de la Seguridad y Salud en el Trabajo. Requisitos con Orientación para su Uso, que indica la necesidad de brindar acceso oportuno a información clara, comprensible y pertinente sobre el sistema de gestión de Seguridad y Salud del Trabajo (ONN, 2018). Igualmente, la Ley 81/97 de la ANPP "Del Medio Ambiente" establece el acceso adecuado a la información sobre medio ambiente que posean los órganos y organismos estatales.

Sobre los requisitos de migración, en las regulaciones archivísticas se advirtieron algunos requisitos sólo en la R. 41/09 del CITMA, entre los que cabe destacar el establecimiento de una política de migraciones periódicas y el diseño de un calendario de revisión y migración. Respecto a las no archivísticas, en el DL. 370/18 se hace referencia a requisitos de migración hacia plataformas de código abierto y de producción nacional. En todos los casos se refiere a la migración tecnológica (por ejemplo, hacia los estándares de 4G, de la red de interconexión de las radiobases a la tecnología IP, etc.).

De tal modo, se pudo constatar que las regulaciones archivísticas y no archivísticas son débiles en lo que respecta a la definición de requisitos para la migración de documentos. Esto implica riesgos para las instituciones de la administración pública cubana, los cuales tienen que ver con la posibilidad de modificación en la codificación binaria de los documentos durante la migración, al punto de que afecte de manera sustancial su capacidad para ofrecer evidencia veraz de los actos y transacciones de las que son reflejo. 


\section{Relación entre las regulaciones archivisticas y no archivisticas}

Existe un reconocimiento por parte del gobierno de la necesidad de contar con sistemas de información más responsables. Lo anterior se adiciona a la necesidad de una AP más transparente para que los ciudadanos tengan la posibilidad de intervenir en la gestión de gobierno y asegurar un mayor control social.

El DL. 281/11 "Del Sistema de Información del Gobierno" establece, entre sus principios, la relevancia, oportunidad y calidad de la información que se gestiona, en particular su veracidad. El Reglamento de la Ley 107/11 "De la Contraloría General de la República de Cuba" plantea un grupo de deberes de los diferentes órganos, organismos, organizaciones y entidades para diseñar, implementar, perfeccionar y verificar la efectividad de un sistema de control interno, entre ellos cabe resaltar el establecimiento de sistemas que garanticen la confiabilidad y oportunidad de la información. En consonancia, la R. 60/11 de la CGR se refiere a la necesidad de disponer de información oportuna y confiable, así como a la rendición transparente de cuentas.

Todo ello implica desarrollar SGD que garanticen información veraz por medio de sus procesos y herramientas. Sin embargo, desde las regulaciones archivísticas no existe una clara visión de cómo la gestión documental interviene en tales desafíos. El DL. 265/09 aborda la práctica archivística desde un marcado enfoque patrimonial. Aunque define la GD, luego no desarrolla esta noción, ni queda claro cómo estos sistemas contribuyen con aspectos como la transparencia y la rendición de cuentas, entre otros.

Resultó llamativo que algunas regulaciones afines como la Ley 1/77 "Ley de Protección al Patrimonio Cultural", el DL. 199/99, el DL. 227/02 "Del Patrimonio Estatal”, el DL. 265/09 y el DL. 281/11 no se relacionan de manera explícita unas con otras a pesar de que coinciden en el mismo objeto, ya que el documento puede ser al mismo tiempo información relevante para una organización, patrimonio estatal, tener valor como patrimonio cultural y ser tratado como información restringida o clasificada.

En las regulaciones no archivísticas analizadas no se hace referencia al DL. 265/09 del CE, salvo en un solo caso: la ya mencionada R. 54/05 del MFP para la definición de los plazos de retención de los estados financieros y sus memorias. Esto puede estar relacionado con el hecho de que el Decreto Ley no está alineado con los requisitos de rendición de cuentas y transparencia que se impulsan en la actual administración pública. 


\section{Enfoque sobre la práctica profesional de gestión documental (inclusiva o exclusiva de la administración de archivo)}

Se pudo apreciar en las fuentes revisadas un marcado enfoque de segmentación profesional entre gestión documental y administración de archivo. El análisis abarcó seis calificadores de cargo del MTSS (R. 3/09, R. 14/09; R. 49/09; R. 75/09; R. 76/09; R. 77/09). Por una parte, aparecen cargos como gestor de archivo central, técnico en gestión documental y especialista en gestión documental; por otra, aparecen especialista en archivística, especialista en procesos archivísticos y especialista en servicios archivísticos. Sin embargo, no existe una diferencia sustancial en cuanto a las funciones que se definen para ambos casos.

Esa visión dicotómica está relacionada con un enfoque, por parte de las autoridades archivísticas cubanas, sobre el documento basado en las perspectivas más tradicionales del ciclo de vida y en la teoría de los valores de Schellenberg (1956), que establece una segmentación entre record y archive. Esto se pudo constatar en el Manual de procedimientos para el tratamiento documental (Marchena et al., 2008) que utiliza el Sistema Nacional de Archivos de la República de Cuba.

Lo anterior dista de los actuales enfoques archivísticos que abogan por una perspectiva holística y de integración sobre la gestión documental, ya sea desde las asunciones más contemporáneas del ciclo de vida, a partir del modelo de cadena de preservación de InterPARES 2 (Eastwood et al., 2008), como desde el modelo de la continuidad de los documentos formulado por Upward $(1996 ; 1997)$.

Una práctica de gestión documental fragmentada impacta de manera desfavorable sobre la gestión de documentos archivísticos electrónicos, que requieren de un tratamiento holístico y de integración profesional ante la dificultad de mantener y evaluar su autenticidad a largo plazo.

\section{Conclusiones}

El marco regulatorio y normativo juega un papel crucial en la manera que las organizaciones gestionan sus documentos archivísticos y generan información confiable, auténtica e íntegra. Con esto se garantiza, además, una gestión pública más consecuente con el modelo de país para 2030.

Del análisis de 322 fuentes legales y normativas cubanas se advirtieron debilidades que dificultan la capacidad de las instituciones de la Administración Pública para gestionar de manera coherente su documentación 
archivística. Este fenómeno conlleva serias implicaciones para la calidad de la información y el desarrollo de procesos de trabajo en entornos que propicien la transparencia, la rendición de cuentas y la seguridad razonable.

Las principales debilidades identificadas se enlistan a continuación:

- Insuficientes requisitos relativos a la gestión de documentos archivísticos. Estos requisitos son de vital importancia para asegurar la veracidad de la documentación archivística en cualquier entorno organizacional.

- Incoherencia entre las políticas de acceso a la información archivística que se establecen para el Sistema Nacional de Archivos de la República de Cuba y otras regulaciones y documentos programáticos del gobierno que promulgan la transparencia y el derecho de los ciudadanos de acceder a la información pública.

- Falta de integración entre las fuentes legales y normativas archivísticas y no archivísticas, lo cual condiciona que los gestores documentales cubanos no se sientan implicados en los desafíos que presenta la AP respecto a la transparencia, la rendición de cuentas, la seguridad de la información, entre otros asuntos.

- Inexistencia de normas cubanas para la gestión documental basadas en buenas prácticas internacionales. De esta manera, los gestores documentales cubanos se ven imposibilitados de aplicar herramientas y métodos que garanticen una actuación más eficiente respecto al tratamiento documental.

- Segmentación profesional entre gestión documental y administración de archivo, lo cual condiciona ineficiencias en la elaboración de estrategias, en la preservación de documentos y falta de integralidad en los profesionales cubanos.

Estos resultados muestran la necesidad de incorporar, en el marco regulatorio y normativo cubano, requisitos coherentes de gestión documental, sobre la base de buenas prácticas internacionales, para que puedan contribuir con el desarrollo de una AP que responda a las demandas de la sociedad cubana actual. 


\section{REFERENCIAS}

ARMA International. 2014. Generally Accepted Recordkeeping Principles. http://www.arma.org/principles.

Bearman, David. 1994. Electronic evidence: strategies for managing records in contemporary organizations. Pittsburgh: Archives \&Museum Informatics.

Cárdenas Travieso, Odalys y Lourdes Margarita Tabares Neira. 2018. "La comunicación y la información en la administración pública”, en Administración pública: conceptos y realidades, editado por Lourdes Margarita Tabares Neyra, 188-216. La Habana: Editorial de Ciencias Sociales.

Committee of Sponsoring Organizations of the Treadway Commission. 2004. Enterprise Risk Management-Integrated Framework. Informe. New York: Institute of Certified Public Accountants.

Constitución de la República de Cuba. 2019. La Habana: Editora Política.

Cook, Terry. 1997. "What is past is prologue: a history of archival ideas since 1898, and the future paradigm shift". Archivaria 43: 17-63.

https://archivaria.ca/index.php/archivaria/article/view/12175

Cook, Terry. 2001. "Archival science and postmodernism: new formulations for old concepts". Archival Science 1 (1): 3-24. https://doi.org/10.1007/BF02435636

Cook, Terry. 2013. "Evidence, memory, identity, and community: four shifting archival paradigms". Archival Science 13 (2): 95-120. https://doi.org/10.1007/s10502-012-9180-7

Dikopoulou, Anastasia y Athanassios Mihiotis. 2011. "The contribution of records management to good governance”. The TQM Journal 24 (2): 123-141.

https://doi.org/10.1108/17542731211215071

Duranti, Luciana. 2001. "The impact of digital technology on archival science". Archival Science 1, 2001: 48. https://doi.org/10.1007/BF02435638

Eastwood, Terry, Bart Ballaux, Rachel Mills y Randy Preston. 2008. "Appendix 14: Chain of Preservation Model - Diagrams and Definitions", en International Research on Permanent Authentic Records in Electronic Systems (InterPARES) 2: Experiential, Interactive and Dynamic Records, editado por Luciana Duranti y Randy Preston, 1-95. Associazione Nazionale Archivistica Italiana. http://www.interpares.org/display_file.cfm?doc=ip2_book_appendix_14.pdf

Evans, Lois, Patricia Franks, Hsuanwei Michelle Chen. 2018. "Voices in the cloud: social media and trust in Canadian and US local governments". Records Management Journal 28 (1): 18-46. https://doi.org/10.1108/RMJ-11-2016-0041

Foscarini, Fiorela. 2010. "Understanding the context of records creation and use: 'Hard' versus 'soft' approaches to records management". Archival Science 10: 389-407. https://doi.org/10.1007/s10502-010-9132-z

Gilliland-Swetland, Anne J. 2000. Enduring paradigm, new Opportunities: the value of the archival perspective in the digital environment. Washington: Council on Library and Information Resources. 
Gilliland-Swetland, Anne J. 2014. "Reconceptualizing records, the archive and archival roles and requirements in a networked society". Knygotyra Book Science 63: 17-34.

https://www.journals.vu.lt/knygotyra/article/view/4011/2773

InterPARES 2 Project. 2008. "The InterPARES 2 Project Glossary”, en International Research on Permanent Authentic Records in Electronic Systems (InterPARES) 2: Experiential, Interactive and Dynamic Records, editado por Luciana Duranti y Randy Preston, 1-50. Roma, Italia: Asociación de Archivística Italiana.

ISO (International Organization for Standardization). 2001a. Information and Documentation - Record management. Part 1: General. ISO 15489-1. Ginebra: ISO.

ISO. 2001b. Information and Documentation. Record Management. Part 2: Guidelines. ISO 15489-2. Ginebra: ISO.

ISO. 2014. Information and Documentation. Risk. Assessment for Records Processes and Systems. ISO 18128. Ginebra: ISO.

ISO. 2016. Information and Documentation. Records Management Part 1: Concepts and Principles. ISO 15489-1. Ginebra: ISO.

Joseph, Pauline, Shelda Debowski y Peter Goldschmidt. 2012. "Paradigm shifts in recordkeeping responsibilities: implications for ISO 15489's implementation”. Records Management Journal 22 (1): 57-75. https://doi.org/10.1108/09565691211222108

Lemieux, Victoria. 2004. "Two approaches to managing information risks". The Information Management Journal 38 (5): 56-62. https://search.proquest.com/docview/227748098/fulltextPDF/411DBD9DD64A4036PQ/1? accountid $=6724$

Lemieux, Victoria. 2010. "The records-risk nexus: exploring the relationship between records and risk". Records Management Journal 20 (2): 199-216. https://doi.org/10.1108/09565691011064331

Marchena, Martha Marina, Olga María Valdés, Marisol León y Mercedes Llovet. 2008. Manual de procedimientos para el tratamiento documental. Santo Domingo, República Dominicana: Archivo General de la Nación.

Marqueti Nodarse, Hiram. 2018. "Gestión pública en Cuba: una aproximación a su situación actual”, en Administración pública: conceptos y realidades, editado por Lourdes Tabares Neyra, 111-148. La Habana: Editorial de Ciencias Sociales.

Mat Isa, Azman. 2009. "Records management and the accountability of governance". Tesis de doctorado, University of Glasgow.

McKemmish, Sue. 2017. "Recordkeeping in the continuum: an Australian tradition", en Research in the Archival Multiverse, editado por Anne Gilliland-Swetland, Sue McKemmish y Andreu J. Lau, 122-160. Australia: Monash University Publishing.

McKinnon, James. 1994. “The 'Sports Rorts' Affair: a case study in record-keeping, accountability and media reporting”. New Zealand Archivist 5 (4): 2-20.

Mena Mugica, Mayra Marta y Jorge del Castillo Guevara. 2018. "Integración de los enfoques de gestión documental y gestión de riesgos para el tratamiento de la información como evidencia de actos y transacciones organizacionales”. Revista Cubana de Información en Ciencias de la Salud 29 (2): 1-17.

http://www.acimed.sld.cu/index.php/acimed/article/view/1213/750 
Menne-Haritz, Angelika. 2001. "Access - the reformulation of an archival paradigm”. Archival Science 1:57-82. https://doi.org/10.1007/BF02435639

ONN (Oficina Nacional de Normalización). 2007. Tecnología de la Información - Código de Buenas Prácticas para la Gestión de la Seguridad de la Información. NC ISO/IEC 17799. La Habana: ONN.

ONN. 2018. Sistema de Gestión de la Seguridad y Salud en el Trabajo. Requisitos con Orientación para su Uso. NC ISO 45001. La Habana: ONN.

Partido Comunista de Cuba. 2016. "Plan Nacional de Desarrollo Económico hasta 2030: Propuesta de Visión de la Nación, Ejes y Sectores Estratégicos”. Proyecto aprobado en el $7 \mathrm{mo}$ Congreso del Partido Comunista de Cuba.

Reed, Barbara. 2005. "Reading the records continuum: interpretations and expectations". Archives and Manuscripts 33 (1): 18-43.

https://search.informit.com.au/documentSummary;dn=200601137;res=IELAPA

Rivero Acosta, Rachel. 2017. "Dar en el control un necesario paso de avance". Granma (31 de enero).

http://www.granma.cu/cuba/2017-01-31/dar-en-el-control-un-necesario-pasode-avance-31-01-2017-23-01-22

Ribeiro, Fernanda. 2017. "La era poscustodial: implicaciones en el campo de la ciencia de la información”, en La Archivística y la Ciencia de la Información Documental: autonomía e interdependencias, editado por Miguel Ángel Rendón Rojas, 2337. Ciudad de México: Instituto de Investigaciones Bibliotecológicas y de la Información, UNAM.

Schellenberg, Theodore R. 1956. Modern archives: principles and techniques. 2a. ed. Chicago: University of Chicago.

Tamayo Pineda, Noris y Julio A. Fernández Estrada. 2018. "Administración pública cubana”, en Administración pública: conceptos y realidades, editado por Lourdes Margarita Tabares Neyra, 11-50. La Habana: Editorial de Ciencias Sociales.

Upward, Frank. 1996. "Structuring the records continuum - part one: Postcustodial principles and properties”. Archives and Manuscripts 24 (2): 268-285. https://search.informit.com.au/documentSummary;dn=970505406;res=IELAPA

Upward, Frank. 1997. "Structuring the Records Continuum, Part Two: Structuration Theory and Recordkeeping”. Archives and Manuscripts 25 (1): 10-35.

https://search.informit.com.au/documentSummary; dn=980100005;res=IELAPA

Para citar este texto:

Castillo Guevara, Jorge del. 2021. "Análisis del marco regulatorio cubano sobre requisitos de gestión documental”. Investigación Bibliotecológica: archivonomía, bibliotecología e información 35 (87): 203-226. http://dx.doi.org/10.22201/iibi.24488321xe.2021.87.58348 


\section{Anexo}

\section{Índice de fuentes legales y normativas citadas}

Decreto 118. 1983. "Reglamento para la Ejecucion de la Ley de Proteccion al Patrimonio". Gaceta Oficial. Cuba: Consejo de Ministros.

Decreto Ley 196. 1999. "Sistema de Trabajo con los Cuadros del Estado y el Gobierno”. Gaceta Oficial. Cuba: Consejo de Estado.

Decreto Ley 199. 1999. "Sobre la Seguridad y Protección de la Información Oficial”. Gaceta Oficial. Cuba: Consejo de Estado.

Decreto Ley 227. 2002. "Del Patrimonio Estatal". Gaceta Oficial. Cuba: Consejo de Estado.

Decreto Ley 265. 2009. "Del Sistema Nacional de Archivos de la República de Cuba”. Gaceta Oficial. Cuba: Consejo de Estado.

Decreto Ley 281. 2011. "Del Sistema de Información del Gobierno”. Gaceta Oficial. Cuba: Consejo de Estado.

Decreto 325. 2014. "Reglamento de la Ley de la Inversión Extranjera”. Gaceta Oficial. Cuba: Consejo de Ministros.

Decreto 326. 2014. "Reglamento del Código de Trabajo". Gaceta Oficial. Cuba: Consejo de Ministros.

Decreto 360. 2019. "Sobre el establecimiento de la seguridad de las tecnologías de la informatización y la comunicación y la defensa del ciberespacio nacional". Gaceta Oficial. Cuba: Consejo de Ministros.

Decreto Ley 370. 2018. "Sobre la informatización de la sociedad cubana”. Gaceta Oficial. Cuba: Consejo de Estado.

Ley 1. 1977. "Ley de Protección al Patrimonio Cultural". Gaceta Oficial. Cuba: Asamblea Nacional del Poder Popular.

Ley 62. 1987. "Código penal”. Gaceta Oficial. Cuba: Asamblea Nacional del Poder Popular.

Ley 81. 1997. "Del Medio Ambiente”. Gaceta Oficial. Cuba: Asamblea Nacional del Poder Popular.

Reglamento de la Ley 107. 2011. "De la Contraloría General de la República de Cuba”. Gaceta Oficial. Cuba: Consejo de Estado.

Resolución 1. 2004. "Requisitos para los Sistemas Contables - Financieros soportados sobre las tecnologías de la información”. Gaceta Oficial. Cuba: Ministerio de Finanzas y Precios y Ministerio de la Informática y las Comunicaciones.

Resolución 3. 2009. "Relación de cargos técnicos, grupos de complejidad, nivel de utilización y requisitos de calificación formal del Sistema Nacional de Auditoría”. Gaceta Oficial. Cuba: Ministerio de Trabajo y Seguridad Social.

Resolución 11. 2007. "Pone en vigor los datos de uso obligatorio que se tendrán en cuenta al momento de diseñar los modelos del subsistema de inventarios”. Gaceta Oficial. Cuba: Ministerio de Finanzas y Precios.

Resolución 11. 2012. "Aprobar el cargo de Especialista Superior en Cuadros". Gaceta Oficial. Cuba: Ministerio de Trabajo y Seguridad Social.

Resolución 14. 2009. "Calificador común de Cargos técnicos y servicios del Sistema de la Educación Superior”. Gaceta Oficial. Cuba: Ministerio de Trabajo y Seguridad Social. 
Resolución 20. 2009. "Sobre Faltantes, Pérdidas y Sobrantes de Bienes”. Gaceta Ofcial. Cuba: Ministerio de Finanzas y Precios.

Resolución 29. 2014 "Aprobar los informes, modelos y registros primarios a emplear por las entidades para cumplir lo establecido en el Reglamento del Código”. Gaceta Oficial. Cuba: Ministerio de Trabajo y Seguridad Social.

Resolución 41. 2009. "Lineamientos para la Conservación de las Fuentes Documentales”. Gaceta Oficial. Cuba: Ministerio de Ciencia, Tecnología y Medio Ambiente.

Resolución 49. 2009. "Calificador ramal de cargos técnicos de la Actividad de Ciencia e Innovación Tecnológica”. Gaceta Oficial. Cuba: Ministerio de Trabajo y Seguridad Social.

Resolución 54. 2005. "Elementos generales que deben contener los documentos normativos sobre contabilidad en las entidades, como parte del sistema de control interno”. Gaceta Oficial. Cuba: Ministerio de Finanzas y Precios.

Resolución 60. 2011. "Normas del Sistema de Control Interno". Gaceta Oficial. Cuba: Contraloría General de la República.

Resolución 75. 2009. "Calificador común de Cargos Administrativos”. Gaceta Oficial. Cuba: Ministerio de Trabajo y Seguridad Social.

Resolución 76. 2009. "Calificador común de Cargos de Servicios". Gaceta Oficial. Cuba: Ministerio de Trabajo y Seguridad Social.

Resolución 77. 2009. “Calificador común de Cargos técnicos”. Gaceta Oficial. Cuba: Ministerio de Trabajo y Seguridad Social. 